\title{
Development of MMIC receivers for cosmic microwave background interferometry
}

\author{
Matthew Sieth ${ }^{a, b}$, Judy M. Lau ${ }^{a, b}$, Patricia Voll ${ }^{a, b}$, Sarah Church ${ }^{a, b}$, Pekka Kangaslahtic ${ }^{c}$ Lorene \\ Samoska $^{c}$, Mary Soria ${ }^{c}$, Todd Gaier ${ }^{c}$, Dan Van Winkle ${ }^{d}$, Jeffrey Neilson ${ }^{d}$, Sami Tantawi ${ }^{d}$, Kieran \\ Cleary $^{e}$, Anthony C.S. Readhead ${ }^{e}$ \\ ${ }^{a}$ Stanford University, 382 Via Pueblo Mall, Stanford, CA 94305, USA; \\ ${ }^{b}$ Kavli Institute for Particle Astrophysics \& Cosmology, \\ MS 29, Stanford, CA 94309, USA; \\ ${ }^{c}$ Jet Propulsion Laboratory, California Institute of Technology, \\ 4800 Oak Grove Drive, Pasadena, CA 91109, USA; \\ ${ }^{d}$ SLAC National Accelerator Laboratory, 2575 Sand Hill Road, Menlo Park, CA 94025, USA; \\ ${ }^{e}$ California Institute of Technology, 1200 East California Boulevard, Pasadena, CA 91125, USA;
}

\begin{abstract}
We report on the development of some of the key technologies that will be needed for a large-format Cosmic Microwave Background (CMB) interferometer with many hundreds of wideband W-band (75-110 GHz) receivers. A scalable threebaseline prototype interferometer is being assembled as a technology demonstration for a future ground- or space-based instrument. Each of the prototype heterodyne receivers integrates two InP Monolithic Microwave Integrated Circuit (MMIC) low-noise amplifiers, a coupled-line bandpass filter, a subharmonic balanced diode mixer, and a $90^{\circ}$ local oscillator phase switch into a single compact module that is suitable for mass production. Room temperature measurements indicate bandaveraged receiver noise temperatures of $500 \mathrm{~K}$ from 85-100 GHz. Cryogenic receiver noise temperatures are expected to be around $50 \mathrm{~K}$.
\end{abstract}

Keywords: Cosmic Microwave Background, polarization, interferometers, mm-wave, MMIC, low-noise amplifiers, largeformat receiver arrays

\section{INTRODUCTION}

The Cosmic Microwave Background (CMB) provides a direct probe of the history of the early universe. Much work has been devoted to studying anisotropies in both the temperature and, more recently, the polarization of the CMB, both of which hold a wealth of information about cosmological parameters. ${ }^{1,2}$ The patterns in polarization maps of the CMB can be decomposed into E-modes, which originate primarily from density fluctuations in the early universe, and B-modes, which are expected to arise from both primordial gravitational waves produced during inflation and gravitational lensing of Emodes. The Degree Angular Scale Interferometer (DASI) ${ }^{3}$ was the first to discover E-mode polarization in the CMB, ${ }^{4}$ and a number of experiments have since probed the E-mode signal in more detail. ${ }^{5-13}$ The next generation of CMB experiments aims to discover primordial B-mode polarization which would in turn provide evidence for inflation via a gravitational wave signature.

Described in this paper are developments towards a prototype W-band B-mode interferometer, which will be scalable to many hundreds of receivers. Section 2 delineates the motivation for developing this technology. The requirements for a large-format interferometer are given in Section 3. These requirements motivate the prototype design, which is discussed in detail in Section 4.

Further author information: (Send correspondence to M.S.)

M.S.: E-mail: mmsieth@stanford.edu, Telephone: 16507259796

Millimeter, Submillimeter, and Far-Infrared Detectors and Instrumentation for Astronomy V, edited by Wayne S. Holland, Jonas Zmuidzinas, Proc. of SPIE Vol. 7741, 77412I

(C) 2010 SPIE $\cdot$ CCC code: $0277-786 X / 10 / \$ 18 \cdot$ doi: $10.1117 / 12.857830$

Proc. of SPIE Vol. 7741 77412I-1 


\section{MOTIVATION}

It is not yet clear whether systematic effects or raw sensitivity will be the limiting factor for the detection of B-modes with future experiments. The systematic errors for aperture plane imagers, such as interferometers, are very different from those of focal plane imagers. One of the principal advantages of interferometers is their ability to reject common-mode noise, which could make some systematic effects easier to control. At the very least, interferometers will provide an independent method for what is a very difficult measurement.

Interferometric techniques have previously been implemented by DASI and the Cosmic Background Imager (CBI) ${ }^{14}$ with much success to study the CMB. However, extending this technique to B-mode measurements has been difficult because of the challenges involved in building the much larger format array that would be required to obtain the necessary sensitivity.

If the curvature of the sky is neglected and the primary beam is assumed to be small, then the response of an interferometer can be approximated as the measurement of the Fourier transform of the sky signal convolved with the primary beam. Although this flat sky assumption is not a particularly good approximation for the angular scales of interest for a B-mode experiment, it is sufficient for the purposes of this paper. Given this approximation, the Rayleigh-Jeans sensitivity to a given Fourier component in the temperature distribution is:

$$
\Delta T_{\min }=\frac{T_{\text {sys }}}{\sqrt{2 n_{\text {base }} \Delta \nu \tau}}
$$

where $\Delta T_{\min }$ is the equivalent temperature of the minimum detectable signal, $T_{\text {sys }}$ is the system noise temperature of a receiver, which is the sum of the receiver noise temperature and atmospheric contributions to the system noise, $n_{\text {base }}$ is the number of correlated baselines that measure a given Fourier mode, $\Delta \nu$ is the bandwidth of each receiver, and $\tau$ is the integration time for the observation. Note that the factor of $\sqrt{2}$ in Equation 1 arises because each pixel is equipped to measure both orthogonal polarization states.

A high sensitivity interferometer requires large quantities of low-noise, wide bandwidth receivers to increase the number of baselines as indicated by Equation 1. DASI and CBI each had 13 single-polarization receivers, each with $10 \mathrm{GHz}$ of bandwidth, that were assembled from discrete components. Future instruments will require hundreds to thousands of dualpolarization detectors with tens of $\mathrm{GHz}$ bandwidth. The requirement for large numbers of detectors necessitates receivers that are mass-producible and highly scalable.

The technology necessary to enable such a large-format interferometer is now within sight. The receiver technology described herein - including feedhorns and polarizers - is highly integrated, compact, and is scalable to many hundreds of elements. This technology builds upon recent receiver developments achieved in experiments such as the Q/U Imaging ExperimenT (QUIET) ${ }^{15}$ and the MMIC Array Spectrograph (MAS). ${ }^{16}$ Moreover, recent developments in FPGA-based digital correlator technology ${ }^{17}$ promise backends capable of processing tens of $\mathrm{GHz}$ bandwidth for large numbers of baselines.

It is also worth noting that the receiver technology under development is extremely versatile. While the receiver modules were designed specifically for CMB interferometery, they can also be used with little or no modification for other applications that require large-format focal plane arrays such as spectroscopy and earth-sensing.

\section{A LARGE-FORMAT B-MODE INTERFEROMETER}

The requirements for a large-format B-mode interferometer motivate the design of the prototype sub-array that is discussed in detail in Section 4. These requirements are listed in Table 1. Large numbers of receivers and wide bandwidth provide high system sensitivity. The primary science band for the full instrument would have a center frequency of $\sim 90 \mathrm{GHz}$ because of the low foreground contamination from synchrotron radiation and dust emission. Note that the full instrument would include receivers with the technology scaled to lower frequencies in order to subtract residual foregrounds based on their spectral signature. In order to probe angular scales at which the expected B-mode signal is strongest, a minimum baseline of $7 \lambda$ at $90 \mathrm{GHz}$ was chosen, corresponding to a multipole moment of $\sim 50$. In order to achieve these small baselines the receivers must be close-packed, which requires that the feedhorns, polarizers, and receiver modules must be smaller than $23 \mathrm{~mm}$ in the transverse dimensions. In order to minimize cross talk while retaining high sensitivity, a beamsize of $14^{\circ} \mathrm{FWHM}$ at the center frequency has been chosen. 

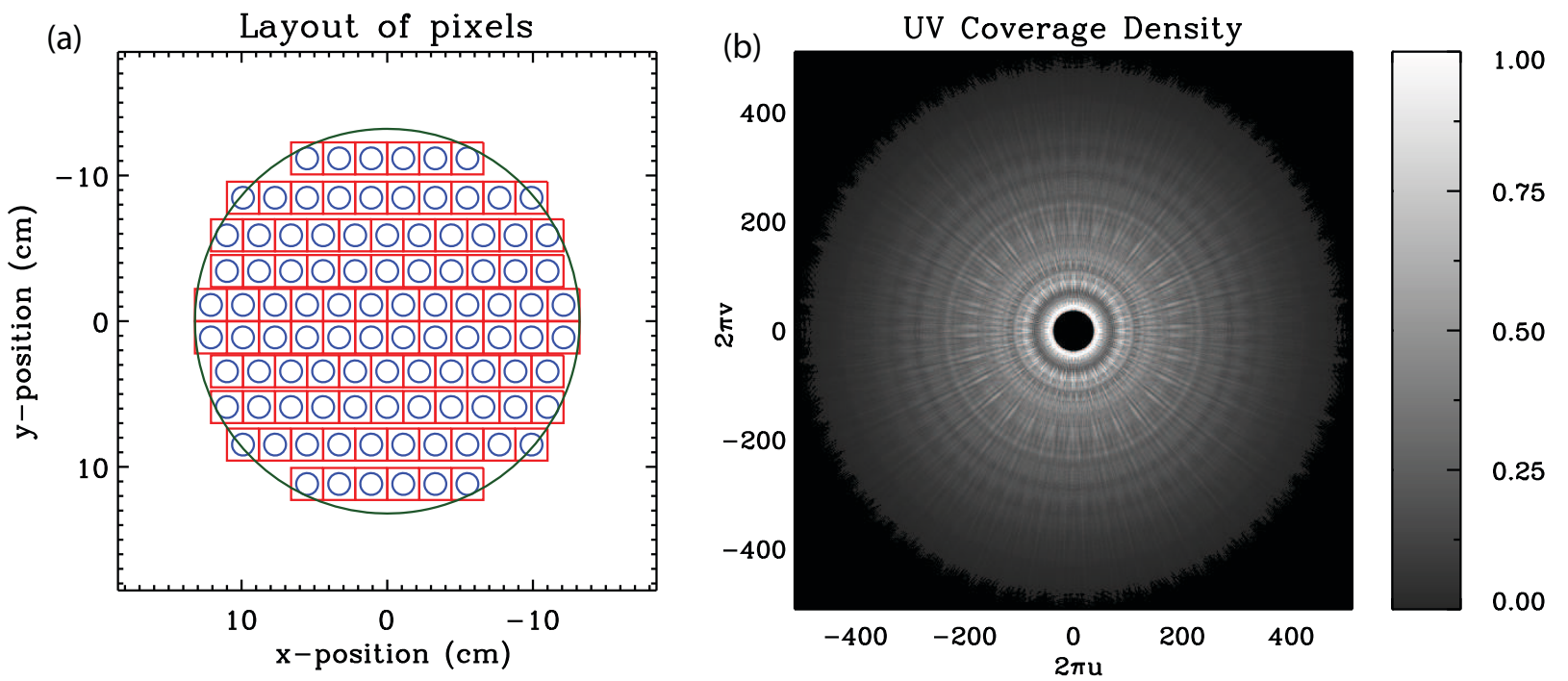

Figure 1. A diagram of the focal plane array for a proposed future CMB interferometer is shown in (a). The small circles represent the feedhorn apertures and the squares are the receiver footprints. The minimum baseline is about $7 \lambda$ at the center frequency, which corresponds to a multipole moment around 50. The corresponding $(u, v)$ coverage is plotted in (b) and is normalized to the maximum coverage density.

Preliminary designs are in place for some of the major components of the large-format instrument. Baselines corresponding to multipole moments $l \sim 50-500$ will be correlated, which covers the range over which the primordial B-mode signal is largest. The receivers will be arranged into cryostats in groups of 100 as shown in Figure 1(a) where the longest baselines correspond to multipoles $\sim 500$. In order to achieve higher sensitivity, several independent cryostats will be built to increase the total number of baselines (see Equation 1). The $(u, v)$ coverage for this arrangement is shown in Figure 1(b) for a bandwidth of $20 \mathrm{GHz}$ and is continuous across $l$-values from 50-500. The incremental increase in array spacing in the $\mathrm{y}$-direction as well as the broad spectral band help to fill in gaps in the $(u, v)$ plane. Note that it would be straightforward to scale the array design to probe higher $l$-values in order to remove B-modes caused by gravitational lensing of the E-mode signal.

The level of B-mode polarization in the CMB depends on the tensor-to-scalar ratio, $r$, which is the ratio of the amplitude of tensor fluctuations - or gravity waves - to that of scalar density perturbations. The expected sensitivity for six 100element arrays in the primary science band, each with $50 \mathrm{~K}$ system temperature, would be sufficient to detect primordial B-mode signals corresponding to $r=0.02$ with 5,000 hours of observation. Lower system temperatures would allow a better limit on $r$.

\begin{tabular}{|c|c|}
\hline \multicolumn{2}{|c|}{ System Requirements } \\
\hline Number of Receivers & 100 per cryostat ${ }^{*}$ \\
\hline Bandwidth & $\sim 20 \mathrm{GHz}$ \\
\hline Center Frequency & $\sim 90 \mathrm{GHz}$ \\
\hline System Temperature & $<50 \mathrm{~K}^{\dagger}$ \\
\hline Beam Size & $14^{\circ} \mathrm{FWHM}$ \\
\hline Polarizations & 2 (RHCP and LHCP) \\
\hline Receiver Footprint & $23 \mathrm{~mm} \times 23 \mathrm{~mm}$ \\
\hline
\end{tabular}

Table 1. Requirements for a large-format B-mode interferometer.

\footnotetext{
* Several cryostats would be used to increase sensitivity as shown in Figure 1.

${ }^{\dagger}$ The system temperature includes noise temperature contributions from both the receiver and the sky.
} 




Figure 2. Schematic showing the signal processing chain for a dual-polarization heterodyne receiver in the prototype interferometer. The output from each feedhorn is split into right- and left-hand polarization components by a waveguide polarizer. The dashed line indicates the extent of the integrated receiver module (see Figure 3). Further amplification and signal processing occurs outside of the module. The second module shown is physically the same as the first, but processes the orthogonal polarization.

\section{PROTOTYPE INTERFEROMETER SUBARRAY}

A three-baseline prototype interferometer is being assembled to demonstrate the low cross talk and scalability that would be required for a future large-scale instrument. A block diagram for the prototype is shown in Figure 2. The output from each feedhorn is split by a waveguide polarizer into left- and right-hand circular polarization components. A compact receiver module contains all of the components necessary to amplify, filter, and downconvert the signal to an intermediate frequency (IF) band. Specifically, 85-100 GHz RF signals are converted to 5-20 GHz IF signals through a single sideband downconversion, which has been implemented in order to reduce the number of high frequency signals to be routed and to simplify the backend signal processing. Further cryogenic amplification of the IF signal occurs outside of the receiver module. A local oscillator (LO) phase-switching scheme is implemented in order to reduce cross talk in the IF signal chain.

\subsection{Housing and Inputs/Outputs}

The use of MMIC technology allows for the integration of the low-noise amplifiers, filter, mixer, and phase switch into a single compact package that is suitable for mass-production. The module housing is machined out of brass and is gold- 
plated to reduce loss. The receiver module housing is made in three parts as shown in Figure 3; the base block holds the receiver components while the other two pieces form lids. The top lid provides a hermetic seal and shields the components from the rest of the system. Such a design is conducive to future automated assembly (the prototype was hand-assembled). The base block and top cover are separately machined such that when they are joined, a WR-10 waveguide input to the module is obtained at the boundary between the two pieces. The components are mounted in 15 mil deep cavities in the base block using silver epoxy $y^{\ddagger}$, which also provides electrical connections to ground.

The LO and IF signals are routed into and out of the module via $1.85 \mathrm{~mm}$ and $2.92 \mathrm{~mm}$ coaxial connectors ${ }^{\S}$, respectively. The connectors are wirebonded to microstrip transmission lines inside the module. The choice of connectors was made for ease of testing of the prototype module with existing lab equipment. Future iterations of the receiver modules will likely employ high frequency push-on connectors, which will enhance the modularity of the system, enabling malfunctioning receivers to be easily replaced.

On the bottom side of the base block, a PC board routes the bias voltages into the receiver. Hermetic DC feedthrus connect the PC board to the top side of the module where the microwave components reside. A miniature 9-pin Microdot connector ${ }^{\|}$attaches to the PC board and is accessible outside the module for easy biasing of the receiver components.

\subsection{Components}

One of the foremost challenges in integrating all of the receiver components is minimizing the system noise. In general, the system noise temperature for a series of $N$ cascaded components is given by:

$$
T_{\text {sys }}=T_{1}+\sum_{i=2}^{N} \frac{T_{i}}{\prod_{j=1}^{i-1} G_{j}}
$$

where $T_{i}$ and $G_{i}$ are the noise temperature and gain, respectively, of the $i^{\text {th }}$ component. If the low-noise amplifiers have sufficient gain, then Equation 2 indicates that the components following the amplifiers contribute negligibly to the system noise temperature. On the other hand, components preceding the amplifiers in general have a non-negligible noise contribution which adds to the total system noise. Additionally, lossy components (i.e. those with $G<1$ ) have a multiplicative contribution that increases the noise contribution of any subsequent components. It is therefore imperative that any component that precedes the low-noise amplifiers have both low loss and noise. In the case where these contributions are minimized, the system noise temperature is dominated by the low-noise amplifiers.

The development of the feeds, polarizers, and receiver modules which integrate the discrete components are described in the following sections. A major emphasis in these designs is to achieve maximum instrument sensitivity (see Equation 1 ), which requires minimizing $T_{\text {sys }}$ while maintaining large bandwidths.

\subsubsection{Feedhorns and polarizers}

To achieve wide-band single mode operation with low return loss and cross talk between pixels, previous instruments have adopted corrugated feedhorn designs ${ }^{18}$ and more recently platelet arrays ${ }^{19}$ because of their excellent spatial response and wide bandwidth. For this application, smooth-walled, spline-profiled feeds are being investigated. These smoothwalled horns can potentially achieve comparable performance to corrugated feedhorns ${ }^{20}$ but are lighter weight and easier to manufacture.

Front-end polarization processing is carried out using a waveguide polarizer in order to minimize loss. The design is a stepped septum polarizer, ${ }^{21}$ based closely on the design used in the QUIET experiment. ${ }^{22}$ Two waveguide outputs, which are linearly polarized, have an amplitude proportional to that of the right- and left-hand circular polarization components at the input.

\footnotetext{
${ }^{\ddagger}$ EPO-TEK H20E

${ }^{\S}$ Anritsu V102F-R \& K102F-R

"Thunderline-Z TL1946

"Tyco USS009C6
} 

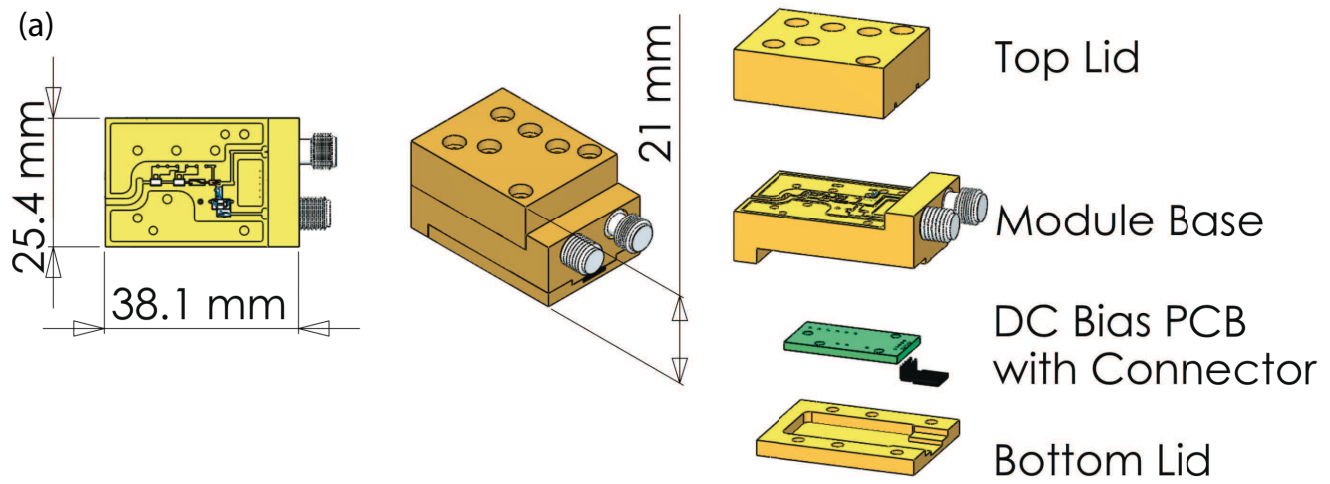

(b)

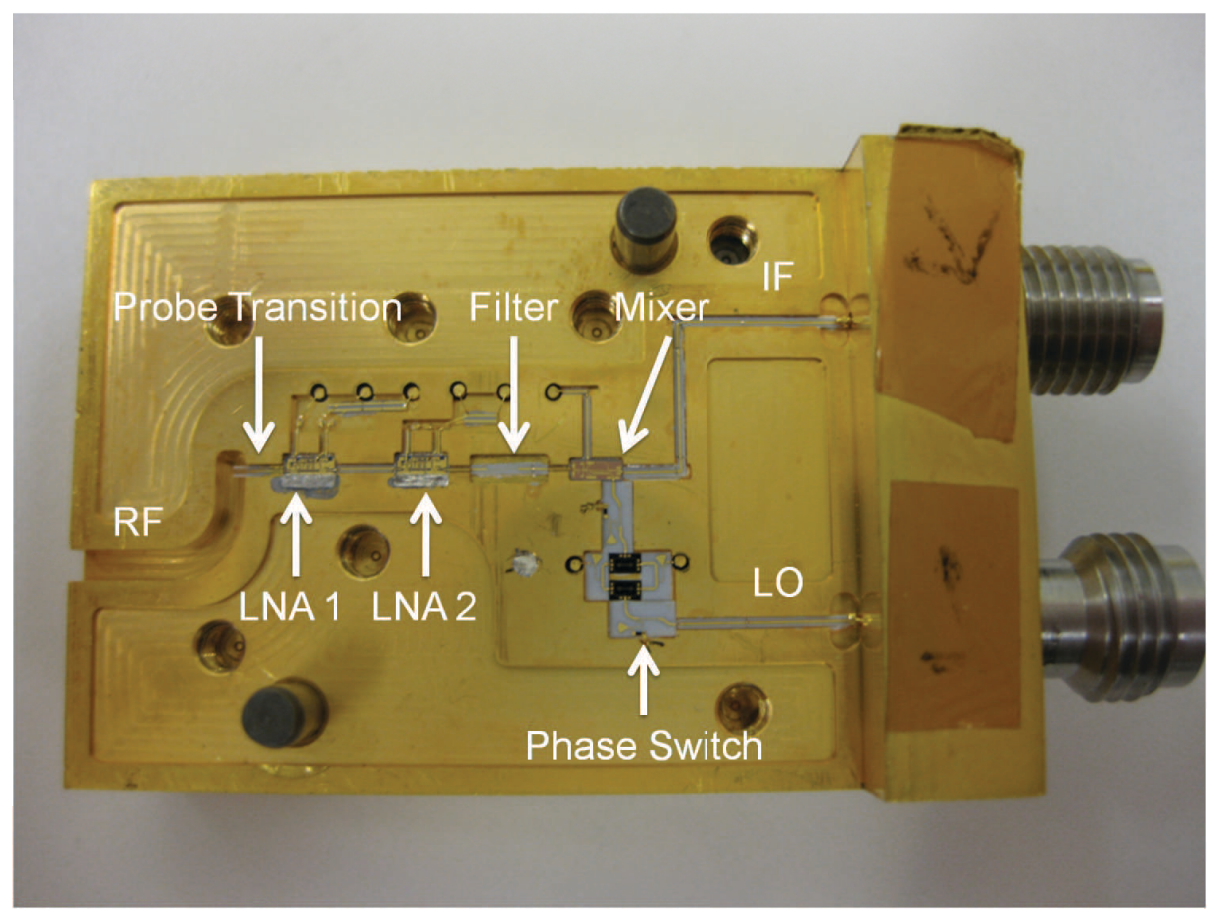

Figure 3. (a) Design concept of the receiver modules. The receiver components are installed in the base plate, which then screws together with the top lid, forming a hermetic seal. A PC board routes DC bias voltages into the module and is enclosed by a bottom lid. (b) Photograph of the interior of one of the prototype receivers with components labeled. Shown are the waveguide-to-planar transmission line transitions, LNAs, filter, mixer phase switch and coaxial connectors. 
(a)



(b)

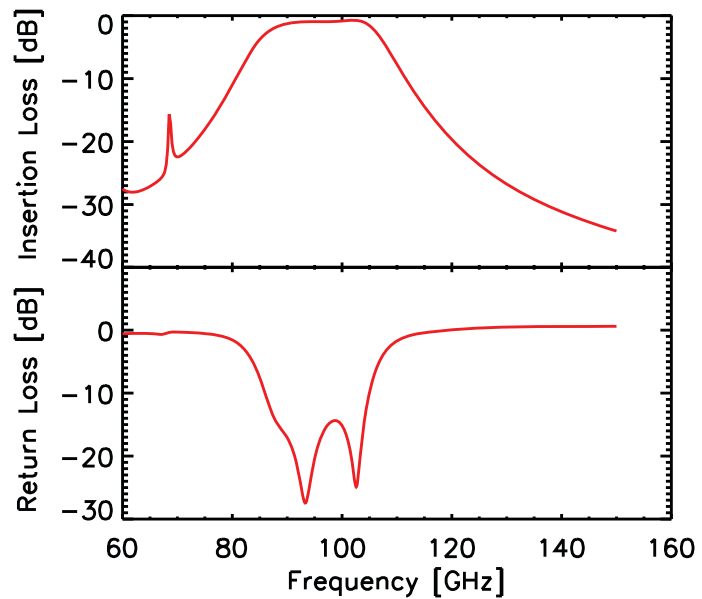

Figure 4. (a) Photograph of a 4-pole coupled-line filter. Chip dimensions are 46 mil x 120 mil. (b) Simulated performance of the filter. The $3 \mathrm{~dB}$ bandwidth is approximately $82-100 \mathrm{GHz}$

\subsubsection{Waveguide-to-planar transmission line transitions}

The low-noise amplifiers are preceded by planar full band waveguide-to-coplanar waveguide (CPW) probe transitions. ${ }^{23}$ The transition, which is patterned on a 4 mil Alumina substrate, is a rectangular probe connected to a section of high impedance microstrip, followed by an impedance matching microstrip quarter-wave transformer and then a microstrip-toCPW transition. As shown in Figure 3, the probe protrudes through an opening in the broadwall of the WR-10 waveguide, oriented along the E-plane of the waveguide (i.e. the plane formed by the direction of the electric field and the direction of propagation). The waveguide also has a quarter-wave backshort, which improves the probe efficiency.

\subsubsection{Low-noise amplifiers}

The signals are amplified by InP MMIC LNAs manufactured by Northrop Grumman Corporation. A combination of 100 $\mathrm{nm}^{24}$ and $35 \mathrm{~nm}^{25}$ gate length InP MMIC LNAs were installed in the receivers. Two configurations of modules have been populated; one has a $35 \mathrm{~nm}$ LNA followed by a $100 \mathrm{~nm}$ LNA and the other has two $100 \mathrm{~nm}$ LNAs.

As discussed in Section 4.2, the receiver sensitivity is expected to be dominated by the noise contributions from the LNAs. The $100 \mathrm{~nm}$ amplifiers are 4-stage InP devices, which typically exhibit band-averaged noise temperatures of $50 \mathrm{~K}$ and gains up to $24 \mathrm{~dB}$ across the $85-105 \mathrm{GHz}$ band at an operating temperature of $24 \mathrm{~K} .{ }^{24}$ These devices are being used in the QUIET experiment. ${ }^{15}$ The $35 \mathrm{~nm}$ amplifiers are 3-stage InP devices and have measured noise temperatures less than $40 \mathrm{~K}$ from $70-96 \mathrm{GHz}^{25}$ with a minimum of $22 \mathrm{~K}$ at $85 \mathrm{GHz}$, while the gain varies from $25-31 \mathrm{~dB}$. Notably, all of these cryogenic measurements were obtained with LNAs installed in waveguide packages and therefore include contributions from the waveguide-to-CPW transitions.

\subsubsection{Filter}

A 4-pole coupled-line bandpass filter is used. The filter is implemented on 4 mil alumina substrate and has a designed $3 \mathrm{~dB}$ bandwidth of 82-100 GHz. This RF filter defines the single sideband downconversion system, passing the upper sideband from the mixer while rejecting the lower sideband. The filter also reduces out-of-band power that might otherwise degrade the system performance by saturating components or by causing resonances in the module. The coupled-line architecture is planar, which makes it easy to integrate into the receiver module. The expected performance, based on simulations in Agilent ADS Momentum, is shown in Figure 4(b).

\subsubsection{Mixer}

The 85-100 GHz RF signal is down-converted to an IF of 5-20 GHz by a subharmonically pumped mixer with a fixed frequency LO of $40 \mathrm{GHz}$. Subharmonic mixers are ideal for this application because the lower frequency LO signal (i.e. half the RF frequency) is easier to route, while still maintaining reasonable conversion loss. The mixer was implemented with an anti-parallel Schottky diode pair using a GaAs process. The conversion loss of the mixer was measured with wafer probes to be 12-17 dB across the band. These results were obtained for a input LO power of $7 \mathrm{dBm}$ and bias of $1 \mathrm{~mA}$. 



(c)

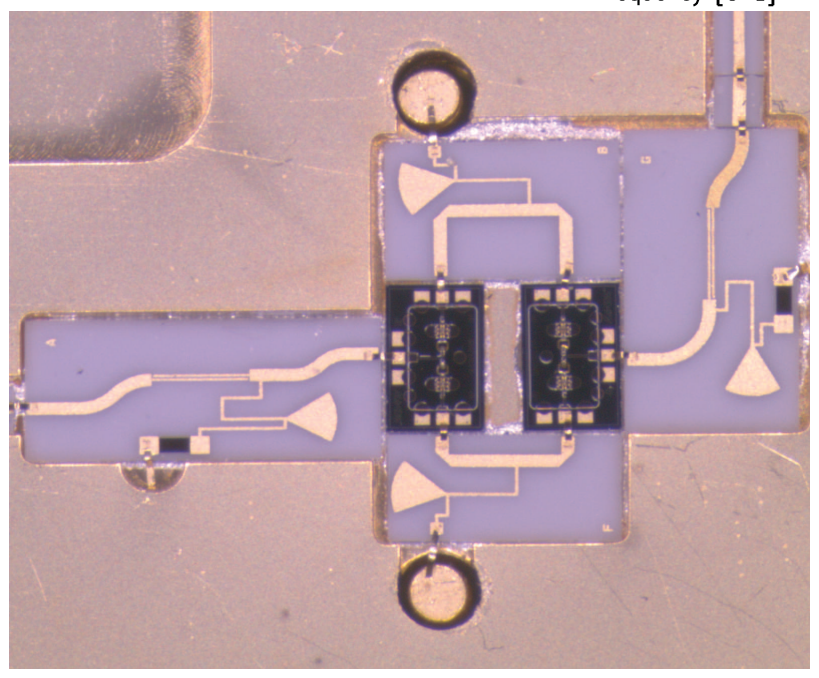

Figure 5. (a) Schematic for the phase switch. The transmission line feeding the LO signal is switched between two microstrip paths, which have a phase difference of $\sim 90^{\circ}$ at $40 \mathrm{GHz}$. (b) Plot of phase shift versus frequency. This iteration of the phase switch has a $93.3^{\circ}$ phase shift at $40 \mathrm{GHz}$. (c) Photograph of the phase switch.

\subsubsection{Phase switch}

Because a second harmonic mixer is being used, the LO signal is phase-switched by $90^{\circ}$ to provide a $180^{\circ}$ switching of the IF signal. Nearby receivers in the interferometer will be phase-switched by orthogonal Walsh functions, which allows for the removal of pixel-to-pixel cross talk that may occur in the IF signal.

The phase switch consists of two AlGaAs SPDT PIN Diode switches**, which switch between two microstrip paths that are designed to have a $90^{\circ}$ phase difference at $40 \mathrm{GHz}$ (see Figures 5(a) and 5(c)). The input and output for the phase switch are capacitively coupled while radial stub capacitors and thin film resistors provide bias circuitry for the SPDT switches. The switching frequency for the prototype is $\sim 10 \mathrm{kHz}$.

The phase switch was measured in a separate test block. The measured phase shift as a function of frequency is shown in Figure 5(b). At the design frequency of $40 \mathrm{GHz}$, the phase switch exhibits $20 \mathrm{~dB}$ return loss and $4.4 \mathrm{~dB}$ insertion loss, while the amplitude imbalance between paths is $0.008 \mathrm{~dB}$. The phase shift is $93.3^{\circ}$ at $40 \mathrm{GHz}$. This will be improved in subsequent designs by appropriate tuning of the circuit based on these measurements.

\subsubsection{IF amplifiers}

Additional IF gain after the receiver modules will be provided by cryogenically cooled amplifiers across the 5-20 GHz band. The choice of IF amplifiers is driven largely by power dissipation. In order to use a single cold-head to cool each 100-receiver cryostat, less than $5 \mathrm{~W}$ of power dissipation from all of the IF amplifiers is desirable. Assuming that two cryogenically cooled amplifiers will be used with each dual-polarization receiver, this leads to a power budget of 25 $\mathrm{mW}$ per IF amplifier. The authors are not aware of any commercial wideband amplifiers that satisfy this specification at cryogenic temperatures. An InP MMIC LNA that has three stages with $100 \mathrm{~nm}$ gate length transistors ${ }^{26}$ has been identified as a candidate for this purpose. If the power budget cannot be met, the cryostat could instead by designed with additional cold heads.

\footnotetext{
${ }^{* *} \mathrm{M} / \mathrm{A}-\mathrm{COM}$ MA4AGSW2
} 


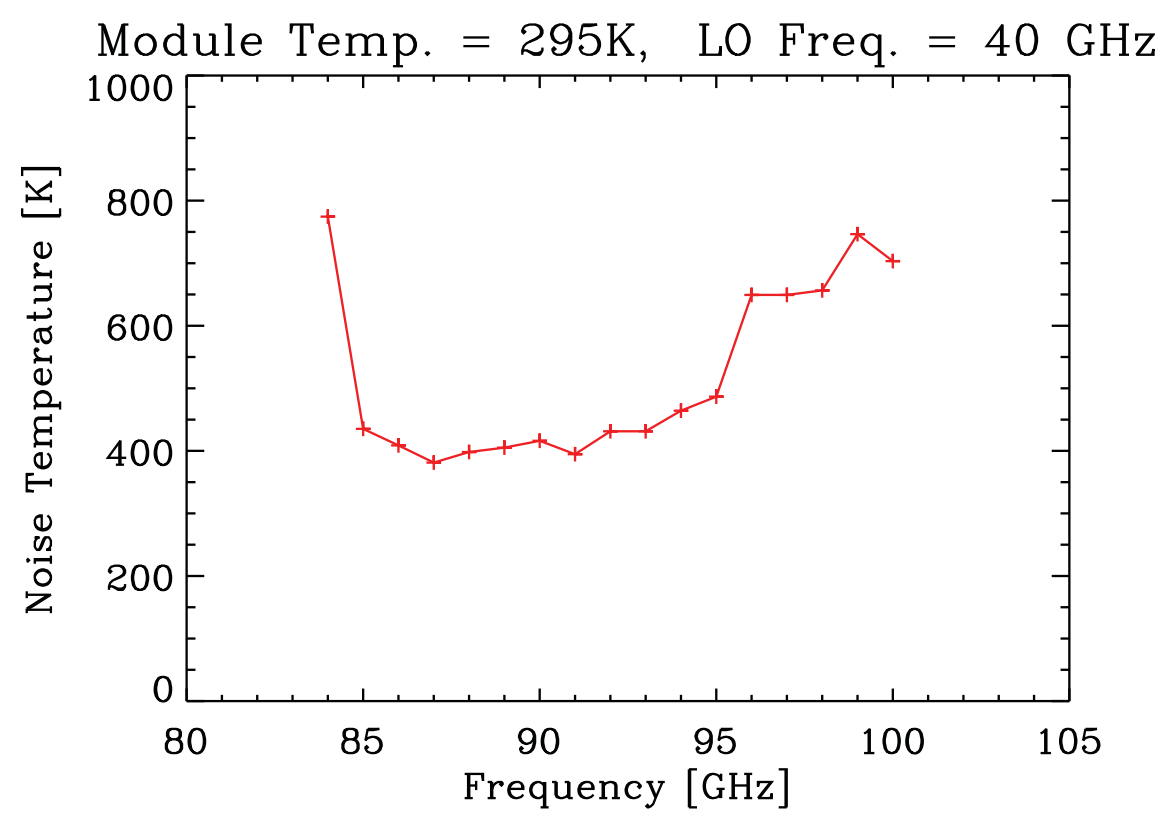

Figure 6. Room temperature noise performance for one of the prototype receivers. The noise temperature is $\sim 500 \mathrm{~K}$ over the $85-100$ $\mathrm{GHz}$ band.

\subsection{System Performance}

Noise temperature measurements have been performed using the standard hot-cold load Y-factor technique. For the noise source, a cone lined with a microwave absorber ${ }^{\dagger \dagger}$ was held at the feedhorn aperture. The hot load was room temperature ( $\sim 295 \mathrm{~K}$ ), while a cold load of $77 \mathrm{~K}$ was achieved by submerging the microwave absorber into liquid nitrogen before performing the measurement. The test configuration does not include the waveguide polarizer, and a commercial WR-10 standard gain pyramidal horn is used to couple the module to the source. Room temperature measurements indicate a band-averaged noise temperatures of $\sim 500 \mathrm{~K}$ across $85-100 \mathrm{GHz}$, as shown in Figure 6 .

Cryogenic measurements are in progress. The expected cryogenic noise performance can be estimated from prior component measurements ${ }^{23-25}$ using Equation 2. The noise that each of the receiver components contributes to the total receiver noise temperature at $90 \mathrm{GHz}$ is summarized in Table 2, where the numbers in parentheses correspond to an LNA fabricated using a newer $35 \mathrm{~nm}$ process as described in [25].

\begin{tabular}{|l|r|}
\hline Component & $\begin{array}{r}\text { Expected Contribution to } T_{\text {sys }} \\
\left(T_{\text {phys }}=20 \mathrm{~K}\right)\end{array}$ \\
\hline \hline Waveguide-to-CPW Transition & $2.2 \mathrm{~K}(2.2 \mathrm{~K})$ \\
\hline LNA 1 & $50.0 \mathrm{~K}(30.0 \mathrm{~K})$ \\
\hline LNA 2 & $0.50 \mathrm{~K}(0.04 \mathrm{~K})$ \\
\hline Mixer & $<0.01 \mathrm{~K}(<0.01 \mathrm{~K})$ \\
\hline \hline Receiver Noise Temperature & $52.7 \mathrm{~K}(32.2 \mathrm{~K})$ \\
\hline
\end{tabular}

Table 2. Projected cryogenic receiver noise temperatures at $90 \mathrm{GHz}$ from individual component data given in [23-25]. The numbers in parentheses correspond to LNAs made using a newer process as described in [25].

\section{CONCLUSIONS AND FUTURE DIRECTIONS}

Highly integrated heterodyne MMIC receiver modules have been developed for use in a three baseline prototype CMB interferometer. These modules are designed to be scaled to a large-format instrument with many hundreds of detectors.

\footnotetext{
${ }^{\dagger \dagger}$ Emerson \& Cumming ECCOSORB
} 
Noise temperatures of $500 \mathrm{~K}$ have been demonstrated at room temperature across the $85-100 \mathrm{GHz}$ band. Cryogenic testing is underway. The noise performance at $20 \mathrm{~K}$ is projected to be $50 \mathrm{~K}$ or below.

In parallel with this effort, work is in progress to develop the following components for completion of the prototype instrument:

- PC boards made on high-frequency laminate are being developed for routing, splitting, and amplifying hundreds of high frequency signals.

- The development of a means to transfer signals across thermal breaks for hundreds of signals.

- High frequency push-on connectors ${ }^{\ddagger \ddagger}$ are being investigated as a future alternative to coaxial connectors. The small size of these connectors facilitates more compact receiver modules. They are also easy to connect and disconnect, which allows for faulty receiver modules to be easily unplugged and replaced.

\section{ACKNOWLEDGMENTS}

The authors would like to thank Matt Morgan for providing the mixer design that was used in the prototype receivers, Eric Bryerton for designing one of the LNAs, Keith Grainge, Andrew Harris, Michael Jones, Tim Pearson, and Angela Taylor for useful discussions about interferometry, Glen Nixon, Suzanne Staggs, and Ed Wollack for useful dialogue about split block polarizers, Dan Werthimer for discussions on correlators, Heather Owen for invaluable help in assembling receiver components, and Rodrigo Reeves for discussions on noise measurements.

This work was supported by the National Aeronautics and Space Administration Stategic University Research Partnership and the SLAC Laboratory Directed Research and Development Program. This work was supported by Department of Energy contract DE-AC03-76SF00515. P.V. thanks the Harriett G. Jenkins Pre-doctoral Fellowship Program for their support.

\section{REFERENCES}

[1] Hu, W., Sugiyama, N., and Silk, J., "The physics of microwave background anisotropies," Nature 386, 37-43 (Mar. 1997).

[2] Komatsu, E., Smith, K. M., Dunkley, J., Bennett, C. L., Gold, B., Hinshaw, G., Jarosik, N., Larson, D., Nolta, M. R., Page, L., Spergel, D. N., Halpern, M., Hill, R. S., Kogut, A., Limon, M., Meyer, S. S., Odegard, N., Tucker, G. S., Weiland, J. L., Wollack, E., and Wright, E. L., "Seven-Year Wilkinson Microwave Anisotropy Probe (WMAP) Observations: Cosmological Interpretation," ArXiv e-prints (Jan. 2010).

[3] Leitch, E. M., Pryke, C., Halverson, N. W., Kovac, J., Davidson, G., LaRoque, S., Schartman, E., Yamasaki, J., Carlstrom, J. E., Holzapfel, W. L., Dragovan, M., Cartwright, J. K., Mason, B. S., Padin, S., Pearson, T. J., Readhead, A. C. S., and Shepherd, M. C., "Experiment Design and First Season Observations with the Degree Angular Scale Interferometer," ApJ 568, 28-37 (Mar. 2002).

[4] Kovac, J. M., Leitch, E. M., Pryke, C., Carlstrom, J. E., Halverson, N. W., and Holzapfel, W. L., "Detection of polarization in the cosmic microwave background using DASI," Nature 420, $772-787$ (Dec. 2002).

[5] Montroy, T. E., Ade, P. A. R., Bock, J. J., Bond, J. R., Borrill, J., Boscaleri, A., Cabella, P., Contaldi, C. R., Crill, B. P., de Bernardis, P., De Gasperis, G., de Oliveira-Costa, A., De Troia, G., di Stefano, G., Hivon, E., Jaffe, A. H., Kisner, T. S., Jones, W. C., Lange, A. E., Masi, S., Mauskopf, P. D., MacTavish, C. J., Melchiorri, A., Natoli, P., Netterfield, C. B., Pascale, E., Piacentini, F., Pogosyan, D., Polenta, G., Prunet, S., Ricciardi, S., Romeo, G., Ruhl, J. E., Santini, P., Tegmark, M., Veneziani, M., and Vittorio, N., "A Measurement of the CMB EE Spectrum from the 2003 Flight of BOOMERANG,” ApJ 647, 813-822 (Aug. 2006).

[6] Sievers, J. L., Achermann, C., Bond, J. R., Bronfman, L., Bustos, R., Contaldi, C. R., Dickinson, C., Ferreira, P. G., Jones, M. E., Lewis, A. M., Mason, B. S., May, J., Myers, S. T., Oyarce, N., Padin, S., Pearson, T. J., Pospieszalski, M., Readhead, A. C. S., Reeves, R., Taylor, A. C., and Torres, S., "Implications of the Cosmic Background Imager Polarization Data," ApJ 660, 976-987 (May 2007).

\footnotetext{
${ }^{\ddagger \ddagger}$ Corning Gilbert GPPO Interconnect Series
} 
[7] Barkats, D., Bischoff, C., Farese, P., Gaier, T., Gundersen, J. O., Hedman, M. M., Hyatt, L., McMahon, J. J., Samtleben, D., Staggs, S. T., Stefanescu, E., Vanderlinde, K., and Winstein, B., "Cosmic Microwave Background Polarimetry Using Correlation Receivers with the PIQUE and CAPMAP Experiments," ApJS 159, 1-26 (July 2005).

[8] Leitch, E. M., Kovac, J. M., Halverson, N. W., Carlstrom, J. E., Pryke, C., and Smith, M. W. E., "Degree Angular Scale Interferometer 3 Year Cosmic Microwave Background Polarization Results," ApJ 624, 10-20 (May 2005).

[9] Larson, D., Dunkley, J., Hinshaw, G., Komatsu, E., Nolta, M. R., Bennett, C. L., Gold, B., Halpern, M., Hill, R. S., Jarosik, N., Kogut, A., Limon, M., Meyer, S. S., Odegard, N., Page, L., Smith, K. M., Spergel, D. N., Tucker, G. S., Weiland, J. L., Wollack, E., and Wright, E. L., "Seven-Year Wilkinson Microwave Anisotropy Probe (WMAP) Observations: Power Spectra and WMAP-Derived Parameters," ArXiv e-prints (Jan. 2010).

[10] Bischoff, C., Hyatt, L., McMahon, J. J., Nixon, G. W., Samtleben, D., Smith, K. M., Vanderlinde, K., Barkats, D., Farese, P., Gaier, T., Gundersen, J. O., Hedman, M. M., Staggs, S. T., and Winstein, B., "New Measurements of Fine-Scale CMB Polarization Power Spectra from CAPMAP at Both 40 and 90 GHz," ApJ 684, 771-789 (Sept. 2008).

[11] Wu, J. H. P., Zuntz, J., Abroe, M. E., Ade, P. A. R., Bock, J., Borrill, J., Collins, J., Hanany, S., Jaffe, A. H., Johnson, B. R., Jones, T., Lee, A. T., Matsumura, T., Rabii, B., Renbarger, T., Richards, P. L., Smoot, G. F., Stompor, R., Tran, H. T., and Winant, C. D., "MAXIPOL: Data Analysis and Results," ApJ 665, 55-66 (Aug. 2007).

[12] Brown, M. L., Ade, P., Bock, J., Bowden, M., Cahill, G., Castro, P. G., Church, S., Culverhouse, T., Friedman, R. B., Ganga, K., Gear, W. K., Gupta, S., Hinderks, J., Kovac, J., Lange, A. E., Leitch, E., Melhuish, S. J., Memari, Y., Murphy, J. A., Orlando, A., O’Sullivan, C., Piccirillo, L., Pryke, C., Rajguru, N., Rusholme, B., Schwarz, R., Taylor, A. N., Thompson, K. L., Turner, A. H., Wu, E. Y. S., Zemcov, M., and The QUa D collaboration, "Improved Measurements of the Temperature and Polarization of the Cosmic Microwave Background from QUaD," ApJ 705, 978-999 (Nov. 2009).

[13] Chiang, H. C., Ade, P. A. R., Barkats, D., Battle, J. O., Bierman, E. M., Bock, J. J., Dowell, C. D., Duband, L., Hivon, E. F., Holzapfel, W. L., Hristov, V. V., Jones, W. C., Keating, B. G., Kovac, J. M., Kuo, C. L., Lange, A. E., Leitch, E. M., Mason, P. V., Matsumura, T., Nguyen, H. T., Ponthieu, N., Pryke, C., Richter, S., Rocha, G., Sheehy, C., Takahashi, Y. D., Tolan, J. E., and Yoon, K. W., "Measurement of Cosmic Microwave Background Polarization Power Spectra from Two Years of BICEP Data," ApJ 711, 1123-1140 (Mar. 2010).

[14] Padin, S., Shepherd, M. C., Cartwright, J. K., Keeney, R. G., Mason, B. S., Pearson, T. J., Readhead, A. C. S., Schaal, W. A., Sievers, J., Udomprasert, P. S., Yamasaki, J. K., Holzapfel, W. L., Carlstrom, J. E., Joy, M., Myers, S. T., and Otarola, A., "The Cosmic Background Imager," PASP 114, 83-97 (Jan. 2002).

[15] Samtleben, D., "Measuring the Cosmic Microwave Background Radiation (CMBR) polarization with QUIET," Nuovo Cimento B Serie 122, 1353-1358 (2007).

[16] Kangaslahti, P., Gaier, T., Cooperrider, J., Samoska, L., Soria, M., ODwyer, I., Weinreb, S., Custodero, B., Owen, H., Grainge, K., Church, S., Lai, R., and Mei, X., "Compact, Miniature MMIC Receiver Modules for an MMIC Array Spectrograph,” NASA Tech. Briefs , 14-15 (Dec. 2009).

[17] Parsons, A., Werthimer, D., Backer, D., Bastian, T., Bower, G., Brisken, W., Chen, H., Deller, A., Filiba, T., Gary, D., Greenhill, L., Hawkins, D., Jones, G., Langston, G., Lasio, J., Van Leeuwen, J., Mitchell, D., Manley, J., Siemion, A., So, H., Whitney, A., Woody, D., Wright, M., and Zarb-Adami, K., "Digital Instrumentation for the Radio Astronomy Community," astro2010: The Astronomy and Astrophysics Decadal Survey 2010, 21-+ (2009).

[18] Gleeson, E., Lanigan, W., Murphy, J. A., O’Sullivan, C., Maffei, B., Church, S. E., and Cartwright, E., "Bandwidth characteristics of corrugated waveguide-horn feeds for CMB experiments," Society of Photo-Optical Instrumentation Engineers (SPIE) Conference Series 5498, 713-724 (Oct. 2004).

[19] Kangas, M. M., Copsey, K., and Lubin, P., "A Modular 100-GHz High-Gain Scalar Corrugated Nonbonded Platelet Antenna," IEEE Antennas and Wireless Propagation Letters 4, 89-92 (Dec. 2005).

[20] Granet, C., James, G. L., Bolton, R., and Moorey, G., "A Smooth-Walled Spline-Profile Horn as an Alternative to the Corrugated Horn for Wide Band Millimeter-Wave Applications," IEEE Transactions on Antennas and Propagation 52, 848-854 (Mar. 2004).

[21] Chen, M. and Tsandoulas, G., "A wide-band square-waveguide array polarizer," IEEE Transactions on Antennas and Propagation 21, 389-391 (May 1973).

[22] Nixon, G., Staggs, S., and Wollack, E. Personal Communication (2009).

[23] Leong, Y.-C. and Weinreb, S., "Full band waveguide-to-microstrip probe transitions," Microwave Symposium Digest, 1999 IEEE MTT-S International 4, 1435 -1438 (1999). 
[24] Weinreb, S., Lai, R., Erickson, N., Gaier, T., and Wielgus, J., "W-band InP wideband MMIC LNA with 30 K noise temperature," Microwave Symposium Digest, 1999 IEEE MTT-S International 1, 101-104 (1999).

[25] Bryerton, E., Mei, X., Kim, Y., Deal, W., Yoshida, W., Lange, M., Uyeda, J., Morgan, M., and Lai, R., “A W-band low-noise amplifier with 22K noise temperature," Microwave Symposium Digest, 2009 IEEE MTT-S International, 681-684 (July 2009).

[26] Rodriguez-Morales, F. and Yngvesson, S., "Integrated THz receivers based on NbN HEB mixers and InP MMIC IF amplifiers," Microwave Symposium Digest, 2005 IEEE MTT-S International , 4 pp. (Dec. 2005). 